\title{
Optimización de sistemas simulados a través de técnicas de superficie de respuesta
}

\author{
Simulated systems optimization through response surface techniques \\ Oscar Oviedo-Trespalacios $^{1} \quad$ Rita Patricia Peñabaena ${ }^{1}$ \\ Recibido 31 de marzo de 2014, aceptado 25 de septiembre de 2014 \\ Received: March 31, $2014 \quad$ Accepted: September 25, 2014
}

\begin{abstract}
RESUMEN
El propósito de este artículo es mostrar la aplicabilidad y ventajas de las técnicas estadísticas de diseño de experimentos como una alternativa útil para la optimización de modelos de simulación discreta. Los sistemas simulados son representaciones computacionales de sistemas reales, se definen como dinámicos debido a que evolucionan en el tiempo por medio de la ocurrencia de eventos discretos, su ventaja es que permiten analizar y experimentar evitando los costos y riesgos que tendría una intervención en un sistema real. En este artículo, mediante un caso de estudio, se desarrolla una metodología que muestra las ventajas de la aplicación de la técnica de superficie de respuesta para la optimización de sistemas simulados. El caso de estudio corresponde a un sistema de producción diseñado con la filosofía empresarial Justo a Tiempo (traducción del inglés Just in Time, JIT), específicamente un sistema KANBAN/CONWIP, donde se analizó el impacto que tienen ciertos factores operacionales acerca de la eficiencia, haciendo uso de técnicas de diseño de experimentos para la optimización estocástica. Los resultados obtenidos demuestran la efectividad que tiene la integración de las técnicas de diseño de experimentos y simulación discreta en la definición de parámetros operacionales de los procesos productivos, además de mejorar la confiabilidad de las metodologías encontradas en la literatura con una nueva consideración estadística.
\end{abstract}

Palabras clave: Diseño de experimentos, sistemas de producción, simulación discreta, superficies de respuesta, optimización.

\begin{abstract}
The purpose of this article is to show the applicability and benefits of the techniques of design of experiments as an optimization tool for discrete simulation models. The simulated systems are computational representations of real-life systems; its characteristics include a constant evolution that follows the occurrence of discrete events along the time. In this study, a production system, designed with the business philosophy JIT (Just in Time) is used, which seeks to achieve excellence in organizations through waste reduction in all the operational aspects. The most typical tool of JIT systems is the KANBAN production control that seeks to synchronize demand with flow of materials, minimize work in process, and define production metrics. Using experimental design techniques for stochastic optimization, the impact of the operational factors on the efficiency of the KANBAN / CONWIP simulation model is analyzed. The results show the effectiveness of the integration of experimental design techniques and discrete simulation models in the calculation of the operational parameters. Furthermore, the reliability of the methodologies found was improved with a new statistical consideration.
\end{abstract}

Keywords: Design of experiments, production systems, discrete simulation, response surface, optimization.

\footnotetext{
1 Departamento de Ingeniería Industrial. Universidad del Norte. Km. 5 vía Puerto Colombia. Barranquilla, Colombia. E-mail: ooviedot@gmail.com; ooviedo@uninorte.edu.co; rpena@uninorte.edu.co
} 


\section{INTRODUCCIÓN}

Con la aplicación de técnicas de simulación discreta y diseño de experimentos en el estudio de sistemas productivos, se ha logrado evaluar independientemente la interacción de las variables del proceso con el objeto de mejorar su eficiencia operativa. Recientemente se ha discutido acerca de la utilidad que brindan las técnicas de diseño de experimentos para el análisis de modelos de simulación, principalmente cuando se cuenta con muchos factores o se quiere analizar si existen iteraciones importantes entre ellos o alguna relación no lineal influyente [1].

Entre las ventajas metodológicas que ofrece la integración del diseño de experimentos y los modelos de simulación discreta está la oportunidad de realizar un análisis de sensibilidad con los parámetros del proceso a diferentes niveles [2-3]. Esto proporciona mayor confianza a la hora de tomar decisiones e incrementa la fiabilidad de las medidas de desempeño de los modelos [4]. No obstante, los recientes avances en el uso de diseño de experimentos con fines de optimización no son frecuentemente utilizados en la literatura especializada, en contraste a los modelos matemáticos y algoritmos exactos/heurísticos con sus limitaciones para determinar los parámetros del proceso [5].

Este artículo toma como base el estudio propuesto por B, Ekren, y A, Örnek [6], con el fin de mejorar su propuesta para la integración de sistemas simulados y técnicas de diseño de experimentos. En [6] se presenta una evaluación de alternativas para aumentar el tiempo de flujo de producción mediante un diseño experimental y simulación discreta. En dicho experimento se analiza y evalúa por medio de un diseño factorial de niveles mixtos el efecto que tienen los parámetros del proceso sobre el desempeño operativo de un sistema manufacturero simulado. Aunque el diseño experimental ejecutado guarda coherencia con el propósito del estudio, es importante notar que como datos de entrada al diseño experimental se utiliza el número total de repeticiones realizadas en cada condición experimental, si bien es posible extraer información de una sola réplica, en una situación típica un programa de simulación se ejecuta $n$ veces con un generador independiente de números aleatorios, estas corridas del modelo de simulación constituyen una muestra aleatoria $\left\{\hat{\theta}_{(1)}, \ldots, \hat{\theta}_{(n)}\right\}$ de estimaciones de $\theta$. Esto implica que cada muestra es idéntica e independientemente distribuida por cada experimento.

En el análisis de los resultados se podría usar entonces estas muestras aleatorias para generar un valor compuesto como estimación de $\theta$, a base de las $n$ réplicas, en orden de incrementar la confiabilidad estadística de la estimación de $\theta$ [7]. La confiabilidad estadística es necesaria para asegurar la validez y precisión de los análisis estadísticos posteriores, por tanto los autores deberían haber utilizado una estimación de las réplicas para cada una de las diferentes condiciones experimentales evaluadas.

Este investigación propone un estudio teóricoexperimental para el desarrollo de una metodología que permita optimizar, con ayuda de técnicas de superficie de respuesta, un proceso productivo simulado, esto con el fin de discutir los supuestos metodológicos encontrados en la literatura y mostrar las ventajas que se obtienen a partir de la aplicación de estas técnicas. Para esto se ha mejorado la confiabilidad estadística de la propuesta de [6], al utilizar un valor compuesto de los resultados de las réplicas de simulación para realizar la optimización.

El caso de estudio utilizado para validar esta propuesta es un sistema elemental de producción Kanban/ CONWIP, que tiene como principal característica que la demanda es la que dirige el flujo de materiales a lo largo del proceso. Este tipo de sistemas se ven motivados por la filosofía Just in Time (JIT), cuyo objetivo es lograr sincronización entre la producción y el mercado [8]. En efecto, este tipo de sistemas permiten el ingreso de materiales a medida que varias señales se emiten como respuesta a cambios de estado en la línea [9]. La diferencia más sutil entre los sistemas jalar y empujar se encuentra en el hecho de que un sistema empujar controla el envío de órdenes de trabajo, mientras que el sistema jalar controla la planta [10-11]. La relevancia de esta aplicación se muestra debido a que existe muy poca literatura que realiza un análisis multifactorial para la implementación y graduación de un sistema de control de la producción tipo jalar, que permita la consecución de una tasa de producción eficiente, para asegurar un excelente nivel de servicio del sistema [12]. 
En la primera parte de este artículo se presenta el marco de experimentación, luego se plantea el modelo de simulación y se fijan posteriormente las condiciones de corridas. El diseño de experimentos se realiza en dos etapas, la primera corresponde al tamizado de factores, para terminar con la construcción de superficies de respuesta y optimización. Al final se presentan las conclusiones.

\section{ANÁLISIS TEÓRICO}

El objetivo de este trabajo es identificar y optimizar los factores que afectan el tiempo de flujo promedio de las unidades fabricadas en un sistema manufacturero Kanban/CONWIP representado mediante un modelo de simulación discreta (ver Figura 1). La metodología utilizada para lograr la optimización del sistema simulado se muestra en la Figura 2.

El primer experimento requirió la definición de varios factores recurrentes en este tipo de sistemas, realizando una revisión de la literatura y consultando a expertos, para concluir en la delimitación de una región factible de operación (ver Tabla 1).

La variable respuesta para este experimento corresponde al tiempo de flujo promedio (ver ecuación (1), que individualmente muestra el tiempo que tarda una unidad de producción en pasar a lo largo de todo un proceso. Donde $\bar{T}_{f}$ corresponde al tiempo de ciclo promedio, $\bar{T}_{0 i}$ y $\bar{T}_{1 i}$ corresponden al tiempo de llegada y salida de la entidad $i$ al sistema y $N$ es el número total de entidades producidas.
Tabla 1. Factores y niveles del experimento

\begin{tabular}{|c|c|c|c|c|}
\hline & Factores & Códigos & Niveles & Unidades \\
\hline & \multirow{3}{*}{$\begin{array}{l}\text { Número de } \\
\text { Tarjetas }\end{array}$} & - (Nivel bajo) & 2 & \multirow{3}{*}{ Tarjetas } \\
\hline & & * (Nivel medio) & 10 & \\
\hline & & + (Nivel alto) & 20 & \\
\hline \multirow{3}{*}{ B } & \multirow{3}{*}{ Capacidad } & - (Nivel bajo) & 1 & \multirow{3}{*}{$\begin{array}{c}\text { Set } \\
\text { Operario- } \\
\text { Máquina }\end{array}$} \\
\hline & & $*($ Nivel medio) & 2 & \\
\hline & & + (Nivel alto) & 3 & \\
\hline \multirow{3}{*}{ C } & \multirow{3}{*}{$\begin{array}{l}\text { Porcentaje } \\
\text { de no } \\
\text { conformes }\end{array}$} & - (Nivel bajo) & $5 \%$ & \multirow{3}{*}{ Porcentaje } \\
\hline & & * (Nivel medio) & $10 \%$ & \\
\hline & & $+($ Nivel alto $)$ & $15 \%$ & \\
\hline \multirow{3}{*}{ D } & \multirow{3}{*}{$\begin{array}{l}\text { Variabilidad } \\
\text { del Sistema }\end{array}$} & - (Nivel bajo) & 0,5 & \multirow{3}{*}{ Minutos } \\
\hline & & $*$ (Nivel medio) & 1 & \\
\hline & & + (Nivel alto) & 1,5 & \\
\hline \multirow{3}{*}{$\mathbf{E}$} & \multirow{3}{*}{$\begin{array}{l}\text { Velocidad } \\
\text { promedio de } \\
\text { producción }\end{array}$} & - (Nivel bajo) & 5 & \multirow{3}{*}{$\begin{array}{c}\text { Minutos } \\
\text { por } \\
\text { Unidad }\end{array}$} \\
\hline & & $*$ (Nivel medio) & 10 & \\
\hline & & $+($ Nivel alto $)$ & 15 & \\
\hline
\end{tabular}

$$
\bar{T}_{f}=\frac{\sum_{i=1}^{N}\left(T_{1 i}-T_{0 i}\right)}{N}
$$

A partir de definir los diferentes factores y la variable respuesta sobre los que se va a experimentar, se procedió a diseñar el modelo de simulación, haciendo uso del software de simulación Arena 12 (C) de Rockwell Automation, como se observa en la Figura 1. El modelo utilizado corresponde al caso elemental del sistema Kanban/CONWIP, semejante a

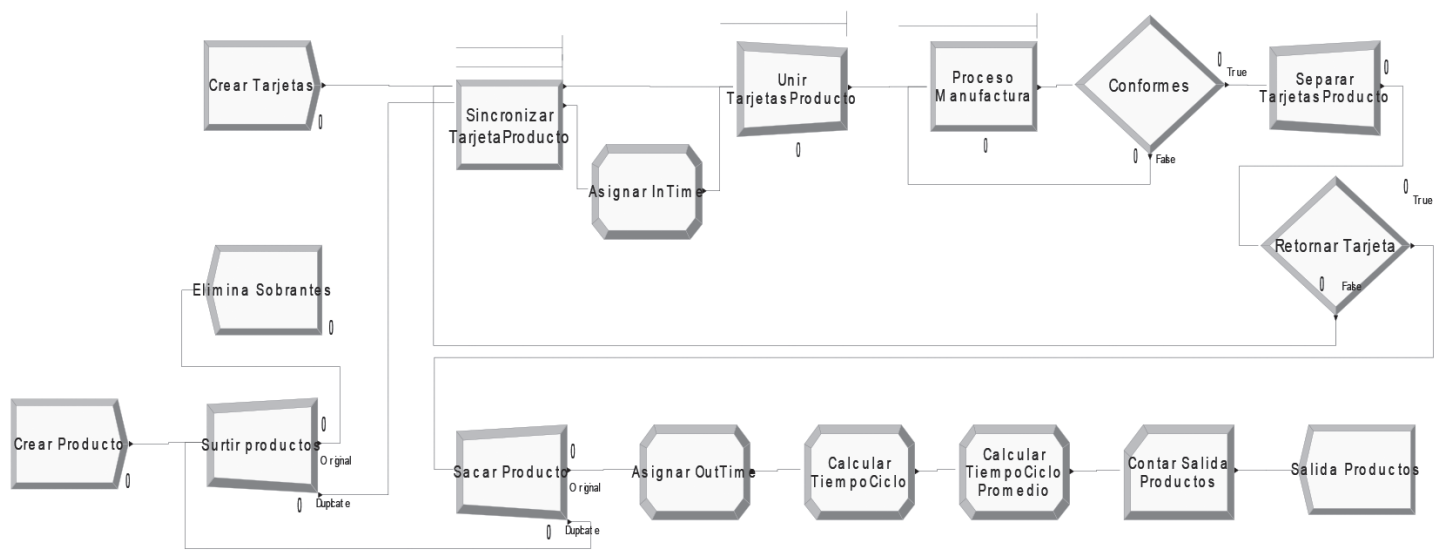

Figura 1. Modelo del sistema Kanban en Arena ${ }^{\circledR}$. 


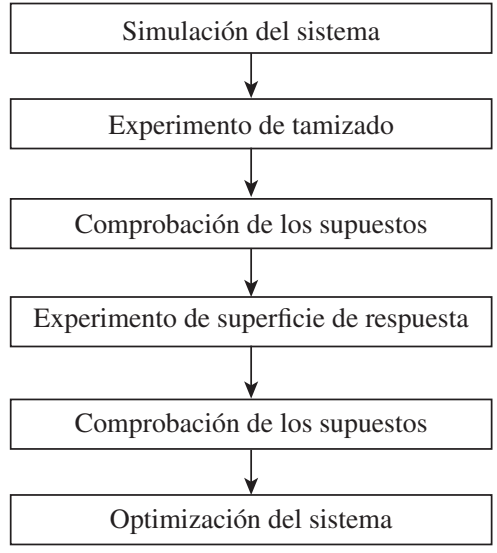

Figura 2. Metodología propuesta.

una estación de trabajo monitoreada con un número de tarjetas determinado de forma manual.

La ejecución del modelo de simulación se realiza 10 veces en cada condición experimental, esto después de verificar que el half-width (valor que proporciona el software Arena para formar un intervalo de confianza, que permita asegurar que el $95 \%$ de las corridas obtenidas se encuentran dentro de los valores formados por la media +- half width o "semirrango") del $T_{f}$ tiempo de flujo promedio fuera lo suficientemente bajo para tener confianza en la información en los resultados del modelo que deben ser ingresados posteriormente al experimento. Cada ejecución del modelo de simulación tiene una duración de 8 horas, en representación de una jornada laboral.

\section{RESULTADOS}

Debido a la cantidad de factores se plantea un experimento de tamizado factorial 25 sin réplicas y 5 puntos centrales, este es un experimento muy común en la industria debido a las grandes ventajas que presenta en términos de economía para realizar el tamizado de factores [13]; debido al nivel de half-width definido del estadístico respuesta y la buena estabilización del sistema, el valor llegó a mantenerse estable y coherente con la confiabilidad deseada en el análisis. La toma de datos se realizó directamente de Arena $12 \AA$ en la medida que se realizaban las modificaciones al modelo y se cumplía con las condiciones de corrida.

Los resultados obtenidos se llevaron a una gráfica de probabilidad normal de los efectos (Figura 3),

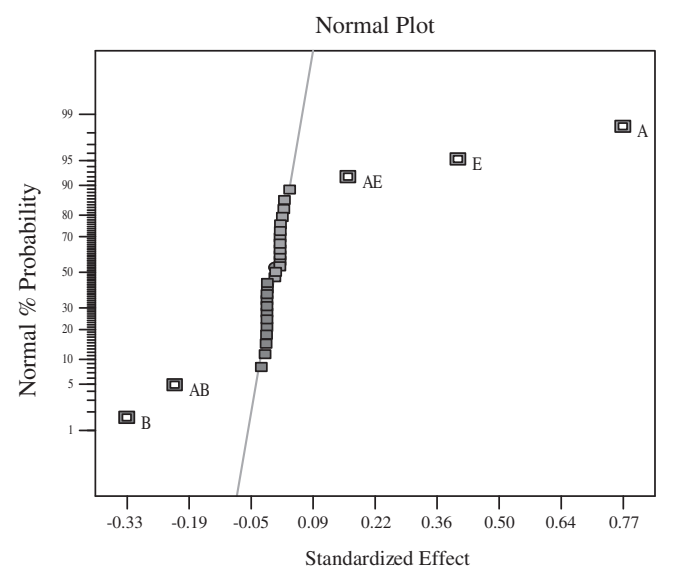

Figura 3. Gráfico de probabilidad normal de los efectos.

que muestra aquellos factores e interacciones que se comportan como una distribución normal y por lo tanto pueden ser considerados error puro. En efecto, solo existen tres factores principales significativos, que corresponde al A-Número de Tarjetas, la B-Capacidad y E-Velocidad de operación del proceso. Adicionalmente el impacto de las AB y $\mathrm{AE}$ debe ser estudiado, pues resultaron significativas. Interacciones de orden superiores fueron rechazadas.

La decisión de incluir puntos centrales permitió tener un estimado del error y realizar un análisis de varianza tradicional, a pesar de tener réplicas en un punto central, el diseño $2^{\mathrm{k}}$ original se mantiene como no replicado, ya que las condiciones experimentales básicas no lo fueron [14]. Los experimentos ejecutados en los puntos centrales nos proveen de un estimado del error puro e información acerca de la presencia de curvatura.

Según los resultados de la Tabla 2, hay suficiente evidencia para asegurar que la curvatura es significativa estadísticamente. Antes de adoptar las conclusiones del análisis de varianza, deberá verificarse la adecuación del modelo fundamental. Para ello inicialmente se realiza el análisis de residuales, donde con ayuda del gráfico de probabilidad normal de los residuos se comprueba que no hay evidencia para creer que los residuos no siguen la distribución normal.

Adicionalmente, con un análisis de los gráficos de residuales contra el número de la corrida se verifica el principio de independencia, esto es esperado 
Tabla 2. ANOVA con análisis de curvatura.

\begin{tabular}{|c|c|c|c|c|c|c|}
\hline Fuente & $\begin{array}{c}\text { Suma de } \\
\text { cuadrados }\end{array}$ & g. 1. & $\begin{array}{l}\text { Cuadrados } \\
\text { medios }\end{array}$ & Valor F & Valor $\mathbf{P}$ & Decisión \\
\hline Modelo & 7,75 & 6 & 1,29 & 2195,38 & $<0,00$ & \\
\hline $\begin{array}{c}\text { A-Número de } \\
\text { tarjetas }\end{array}$ & 4,98 & 1 & 4,98 & 8468,14 & $<0,00$ & \\
\hline B-Capacidad & 0,92 & 1 & 0,93 & 1574,25 & $<0,00$ & \\
\hline E-Velocidad & 1,24 & 1 & 1,24 & 2115,06 & $<0,00$ & \\
\hline $\mathbf{A B}$ & 0,34 & 1 & 0,34 & 569,34 & $<0,00$ & \\
\hline $\mathbf{A E}$ & 0,26 & 1 & 0,26 & 439,11 & $<0,00$ & \\
\hline Curvatura & 0,02 & 1 & 0,02 & 27,18 & $<0,00$ & Significativo \\
\hline Residuales & 0,02 & 29 & 0,00 & & & \\
\hline Falta de ajuste & 0,02 & 25 & 0,00 & & & \\
\hline Error puro & 0,00 & 4 & 0,00 & & & \\
\hline Total & 7,79 & 36 & & & & \\
\hline
\end{tabular}

por tratarse de un modelo de simulación discreta basado en corridas independientes e idénticamente distribuidas. Los gráficos de residuos contra predichos totales y los de cada uno de los factores, muestran que la varianza es consistente para cada uno de ellos y los datos se encuentran entre 3 y -3 veces la desviación estándar, por tanto no se presentan problemas de homocedasticidad.

Para el planteamiento de la superficie de respuesta se planteó un nuevo experimento con diseño de $3^{\mathrm{k}}$. Los factores que fueron retirados en el experimento anterior por su baja significancia se fijaron en las condiciones más bajas de operación utilizando un criterio de costo/beneficio, suponiendo que a medida que operaban en niveles más elevados, requieren una mayor cantidad de recursos.

A partir del diseño propuesto anteriormente se construyó el modelo asociado al proceso, para esto se tuvo en cuenta que el modelo global y todo sus términos sean significativos (ecuación (2)). Igualmente, los residuales resultaron aleatorios afirmando el poder explicativo del modelo. $\mathrm{La}$ ANOVA del modelo de regresión de ajuste a los datos, nos muestra los resultados del análisis de varianza, en esta el modelo global resulta significativo, al igual que cada uno de sus términos del modelo (Tabla 3). Esto se debe a que el valor P se mantiene menor que 0,05 .

Tabla 3. ANOVA del modelo de regresión.

\begin{tabular}{|c|c|c|c|c|c|c|}
\hline Fuente & $\begin{array}{c}\text { Suma de } \\
\text { cuadrados }\end{array}$ & g. 1. & $\begin{array}{c}\text { Cuadrados } \\
\text { medios }\end{array}$ & Valor $\mathbf{F}$ & Valor $\mathbf{P}$ & Decisión \\
\hline Modelo & 5,09 & 7,00 & 0,73 & 176,08 & $<0,0001$ & significativo \\
\hline $\begin{array}{l}\text { A-Número de } \\
\text { tarjetas }\end{array}$ & 3,01 & 1,00 & 3,01 & 729,97 & $<0,0001$ & significativo \\
\hline B-Capacidad & 0,79 & 1,00 & 0,79 & 192,12 & $<0,0001$ & significativo \\
\hline E-Velocidad & 0,89 & 1,00 & 0,89 & 216,56 & $<0,0001$ & significativo \\
\hline $\mathbf{A B}$ & 0,14 & 1,00 & 0,14 & 34,56 & $<0,0001$ & significativo \\
\hline $\mathbf{A E}$ & 0,13 & 1,00 & 0,13 & 30,37 & $<0,0001$ & significativo \\
\hline$A^{\wedge} 2$ & 0,10 & 1,00 & 0,10 & 24,42 & $<0,0001$ & significativo \\
\hline $\mathrm{B}^{\wedge} 2$ & 0,04 & 1,00 & 0,04 & 10,62 & 0,0033 & \\
\hline Residuales & 0,10 & 24,00 & 0,00 & & & \\
\hline Falta de ajuste & 0,10 & 19,00 & 0,01 & & & \\
\hline Error puro & 0,00 & 5,00 & 0,00 & & & \\
\hline Total & 5,19 & 31,00 & & & & \\
\hline
\end{tabular}


$T_{f}=0,27+0,08 \mathrm{x}_{\mathrm{A}}-0,38 \mathrm{x}_{\mathrm{B}}+0,02 \mathrm{x}_{\mathrm{E}}-$

$0,01 \mathrm{x}_{\mathrm{A}} \mathrm{x}_{\mathrm{B}}+0,002 \mathrm{x}_{\mathrm{A}} \mathrm{x}_{\mathrm{E}}-0,001 \mathrm{x}_{\mathrm{A}}^{2}+0,08 \mathrm{x}_{\mathrm{B}}^{2}$

Siendo $\mathrm{x}_{\mathrm{A}}$ : Número de tarjetas, $\mathrm{x}_{\mathrm{B}}$ : Capacidad $\mathrm{y}$ $\mathrm{x}_{\mathrm{E}}$ : Velocidad.

En las gráficas de residuales también se observa que los residuos se ajustan a una distribución normal, no se aprecian problemas de independencia al analizar los residuos contra el orden de corrida, y las gráficas de residuales contra predichos se encuentran entre los límites de -3 y 3 .

Las superficies de respuesta 3D se graficaron con ayuda del software Design Expert ${ }^{\circledR}$. El tiempo mínimo de flujo se minimiza cuando E llega a su nivel más bajo, mientras que la interacción entre A y B presenta la mayor área de niveles bajos de tiempo de flujo promedio (ver Figura 4. Gráfico de superficie de respuesta de $\mathrm{AB}$ con $\mathrm{E}$ en $\mathrm{BAJO}$, que presenta la zona más clara que indica niveles bajos de tiempo de flujo). En la Figura 4 se encuentra que los incrementos de capacidad no modifican considerablemente el tiempo de flujo de promedio, más aun cuando disminuye el número de tarjetas en el sistema.

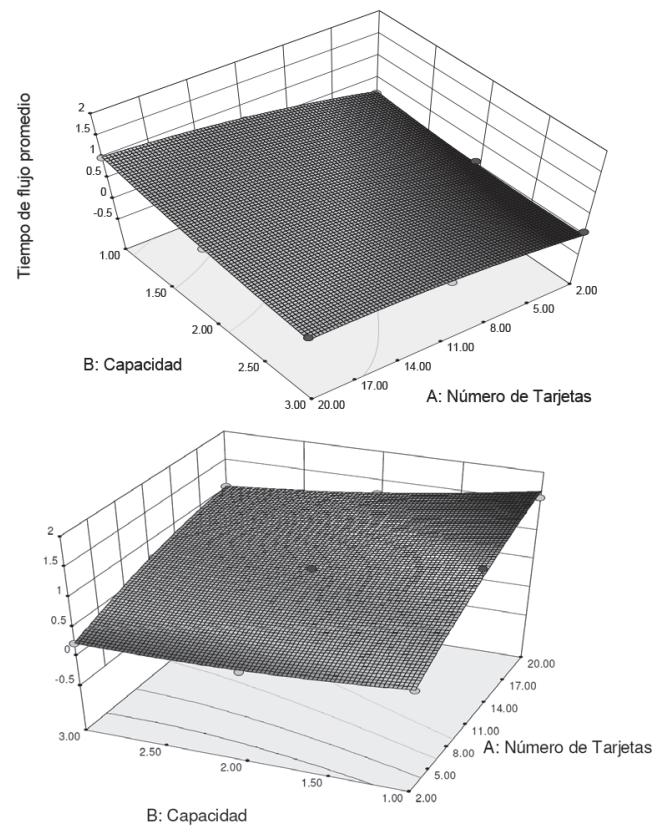

Figura 4. Gráfico de superficie de respuesta de i) $\mathrm{AB}$ con $\mathrm{E}$ en $\mathrm{BAJO}$ y ii) $\mathrm{AB}$ con $\mathrm{E}$ en ALTO.
Los resultados muestran que cuando B se encuentra en su nivel más alto, la interacción entre $\mathrm{A}$ y $\mathrm{E}$ presenta la mayor área de niveles bajos de tiempo de flujo promedio (ver Figura 4), Gráfico de superficie de respuesta de AE con B en ALTO, que presenta la zona más clara indicadora de niveles bajos de tiempo de flujo,

Con el análisis de superficies de respuesta se puede concluir que los sistemas Kanban/CONWIP el número de tarjetas o señales dentro del sistema es concluyente en su desempeño, esto ha sido reportado antes por [9]. Así mismo, la interacción que tiene con otros factores resulta significativa en la determinación del tiempo de ciclo. En definitiva los niveles de una velocidad de producción por unidad BAJA y una capacidad ALTA son condiciones deseadas del sistema, pero no estrictas para conseguir un tiempo de flujo promedio bajo para las unidades del sistema.

Para realizar la optimización del proceso se utilizó Design Expert ${ }^{\circledR}$ y su módulo de optimización estocástica, que trabaja mediante el método desarrollado por [15]. Para esto se debe seleccionar la meta de optimización para cada factor y respuesta, que para el caso específico de este estudio se constituye en la minimización de tiempo de flujo promedio. Adicionalmente se debe mantener la región factible de operación (ver Tabla 4).

La búsqueda empieza en un punto de partida aleatorio y continúa a lo largo de la pendiente más pronunciada hasta que encuentra un óptimo (en este caso busca un mínimo). Posiblemente pueden existir varios puntos óptimos en la superficie debido a la curvatura, en este estudio se evaluaron 57 puntos distintos, utilizando el valor predefinido del paquete de optimización. Los resultados de la optimización muestran que en el punto 28 se logró minimizar el tiempo de flujo promedio a 0,065 , con

Tabla 4. Condiciones de Optimización

\begin{tabular}{|c|c|c|c|}
\hline $\begin{array}{c}\text { Variables y } \\
\text { objetivos }\end{array}$ & Meta & $\begin{array}{c}\text { Límite } \\
\text { inferior }\end{array}$ & $\begin{array}{c}\text { Límite } \\
\text { superior }\end{array}$ \\
\hline $\begin{array}{c}\text { A:Número de } \\
\text { tarjetas }\end{array}$ & En rango & 2 & 20 \\
\hline B:Capacidad & En rango & 1 & 3 \\
\hline E:Velocidad & En rango & 5 & 15 \\
\hline $\begin{array}{c}\text { Tiempo de flujo } \\
\text { promedio }\end{array}$ & Minimizar & 0,08 & 1,57 \\
\hline
\end{tabular}


unas condiciones experimentales de 2,18 tarjetas (A-Número de tarjetas), 2,74 sets hombre/máquina (B-Capacidad) y 7,34 minutos en promedio por unidad (E-Velocidad). Al repetir estas condiciones no se observan cambios en el resultado por encima del 0,001 de flujo promedio

\section{CONCLUSIONES}

En este trabajo se ponen en evidencia las ventajas operacionales que representa el uso de simulación discreta en conjunto con técnicas de diseño de experimentos para la optimización de sistemas, esto debido a que mediante diseños experimentales podemos describir la interacción y relaciones que tienen diferentes factores acerca del sistema. Además, haciendo uso de esta metodología es posible evaluar el efecto de las interacciones o curvaturas de los planos de operación.

La metodología propuesta se puede tomar como referente para la realización de estudios similares, Además los resultados mostrados cumplieron con todos los supuestos necesarios para hacer uso de las herramientas de simulación y diseño de experimentos, esto es algo que no se considera en la literatura científica relacionada del tema. En el caso de estudio se logró consolidar la importancia que tiene sobre el tiempo de flujo promedio de las unidades en un sistema Kanban/CONWIP el número de tarjetas dentro del sistema y además se encontró y describió la forma en que estas se relacionan estrechamente con la capacidad y la velocidad promedio de producción de cada unidad de producto. También se logró descartar la influencia de variables del sistema como el porcentaje de no conformes y la variabilidad del proceso de fabricación.

Se encontró que el modelo que explica la variable respuesta en términos del número de tarjetas, capacidad y la velocidad promedio de procesamiento por unidad, se compone de variables de segundo orden o cuadráticas, a partir de esto se logró hallar la superficie de respuesta y utilizar la técnica de optimización estocástica. Los valores en los que se debe fijar el proceso es 2,18 tarjetas (A-Número de tarjetas), 2,74 sets hombre/máquina (B-Capacidad) y 7,34 minutos en promedio por unidad (E-Velocidad), mientras que los factores eliminados de porcentaje de no conformes (C) y variabilidad del sistema (D) se pueden ubicar en cualquier nivel, con preferencia un criterio económico que consuma menos recursos.

La información que se encontró con este trabajo es muy valiosa para caracterizar las relaciones en este tipo de procesos industriales, además de ofrecer una alternativa para el análisis de este tipo de entornos y las posibilidades que hay en términos de optimización mediante la simulación discreta y el diseño de experimentos. Como futuras investigaciones se propone replicar estas técnicas en la determinación de los parámetros en los sistemas de calidad [16].

\section{REFERENCIAS}

[1] S. Sánchez, F. Moeeni and P. Sánchez. "So many factors, so little time ... Simulation experiments in the frequency domain". International Journal of Production Economics. Vol. 103, pp. 149-165. September, 2006. ISSN: 0925-5273.

[2] J. Kleijnen. "Experimental Design for Sensitivity Analysis of Simulations Models". Proceedings of EUROSIM 2001, 2001.

[3] D. Kelton and R. Barton. "Experimental Design for Simulation". Proceedings of the 2003 Winter Simulation Conference. 2003.

[4] B. Bettonvil and P. Kleijnen. "Identifying the important factors in simulation models with many factors". Tilburg University. Departament of information Systems and Center for Economic Research. Netherlands, 1994.

[5] R. Peñabaena-Niebles, O. Oviedo-Trespalacios, K. Ramírez y M. Morón. "Diseño EconómicoEstadístico de Cartas con Parámetros Variables Totalmente Adaptativas en Presencia de Datos Autocorrelacionados". Revista Iberoamericana de Automática e Informática Industrial RIAI. Vol. 11, pp. 247-255. Abril 2014. ISSN: 1697-7912.

[6] B. Ekren and A. Örnek "A simulation based experimental design to analyze factors affecting production flow time". Simulation Modelling Practice and Theory. Vol. $16 \mathrm{~N}^{\circ} 3$, pp. 278-293. March, 2008. ISSN: 1569-190X.

[7] T. Altiko and B. Melamed. "Simulation Modeling and Analysis with Arena". Academic Press. 2007. 
[8] M. Di Mascolo, Y. Frein and Y. Dallery “An Analytical method for performance evaluation of kanban controlled production systems". Operation Research. Vol. 44, pp. 50-64. January, 1996. ISSN: 0030-364X.

[9] T. Hochreiter. "A comparative simulation study of Kanban, CONWIP, and MRP manufacturing control systems in a flowshop". Master of Science Thesis. University of Florida. Gainesville, USA. 1999.

[10] D. Sipper y R. Bulfin. "Planeación y control de la producción”. Mc Graw Hill. 1998.

[11] M. Spearman and M. Zanzanis. "Push and pull systems: Issues and comparison". Operation Research, Vol. 40, pp. 525-532. May, 1992. ISSN: 0030-364X

[12] M. Lage Junior and M. Godinho Filho. "Variations of the kanban system: Literature review and classification". Int J Prod Econ.
Vol. 125, pp. 13-21. May, 2010. ISSN: 0925-5273.

[13] D.C. Montgomery. "Design and Analysis of Experiments". John Wiley \& Sons, New York. 1996.

[14] Reliasoft. DOE++ User's Guide. ReliaSoft's DOE++ software. 2008. URL: http://www. weibull.com/DOEWeb/

[15] W.R. Myers, D.C. Montgomery and C.M. Anderson-Cook. "Response Surface Methodology". 3rd edition, John Wiley and Sons. New York, USA. 2009.

[16] R. Peñabaena-Niebles, O. OviedoTrespalacios, S. Cuentas-Hernández y E. García-Solano. "Metodología para la implementación del diseño económico y/o estadístico de cartas de control x-barra con parámetros variables (VP)". Dyna. Vol. 81, pp. 150-157. Abril 2014. ISSN: 0012-7353. 